\title{
Index of languages and scripts
}

Ajami $1,11 \mathrm{fn} 23,32,33$

Brzopis 191, 192 fig2b, 193 fig 3b

Burmese (script) $39 \mathrm{fn} 7$

Byzantine Greek 188 fn8, 207

Church Slavonic 185ff., 189 fn10, 194, 194

fn15, 195 fn16, 197ff., 199 fn24, 201, 201

fn27, 202ff., 206, 210 fn 41, 217

Cursive script 191, 192, 192 fig2b, 193 fig3b

Dhamma script $36,36 \mathrm{fn} 2,49,49$ fn 34, 51,

$53,56,58$

Druckminuskel 251, $251 \mathrm{fn} 65$

Gə`əz 271

Kam Müang (Lan Na language) 36

Lao (script) 45, 52, 52 fig6

Latin $25 \mathrm{fn} 47,217,225 \mathrm{fn} 6,226,229,230$, 233, $234 \mathrm{fn} 20,235,235 \mathrm{fn} 21,237 \mathrm{ff}$. , $240 \mathrm{fn} 37,241,242,244,251,253,253$ fn76, 263

Lik Hto Ngouk (Tai Noe script) $49,51,51 \mathrm{fn} 5$

Maiuscola ogivale inclinata 250

Malayalam (language) $60 \mathrm{fn} 4$

Malayalam (script) $60 \mathrm{fn} 4$

Mande languages 1, 2 fn3, 33

Manding (language group) 1, $24 \mathrm{fn} 47,32 \mathrm{f}$.

Mandinka $1 \mathrm{fn} 1,32 \mathrm{f}$.

Manipravalam $62,62 \mathrm{fn} 5+6,63,68,73$
New Tai Lü (script) $\quad 49,50$ fig 4

Old Greek 7, 239

Old Mande 1ff., 22 fn43

Old Mon script 36

Old Tai Lü (script) $\quad 49,50$ fig3

Pali 36,39 fn7, 20 fn10, 42 fn14, 43 fn17, 46, 49 fn34, 51

Sanskrit $43 \mathrm{fn} 17,46 \mathrm{fn} 28,61 \mathrm{f}$., $62 \mathrm{fn} 5+6$, $63,63 \mathrm{fn} 8,68 \mathrm{ff} ., 69 \mathrm{fn} 26,70,72,73,73$ fn41, 74, 74 fn 44, 75, 81 fn56, 84, 85, 113, 115, 121

Semi-uncial script 189, 190 fig1, 191, 191 fig1b, 192 fig2b, 193 fig31

Shan (script) [shani] 51, 51 fig5, 52, 58

Soninke $1 \mathrm{fn} 1,2,2 \mathrm{fn} 2,18,18 \mathrm{fn} 35,22 \mathrm{fn} 43$, 25, 27, 27 fig12, 28 fn 48,29 fn 48,32

Tai Khün (language) 36, 37, 43 fn17, 47, 49

Tai Lü (language) $36,37 \mathrm{fn} 5,38 \mathrm{fn} 6,40 \mathrm{fn} 9$, $42 \mathrm{fn} 15,43 \mathrm{fn} 15+17,49$

Tai Noe (language) $49,51,51$ fig5, 52

Tamil (language) 62, 68f., 69 fn26, 70, 70 fn $31+32+33,71,71$ fn37, $73 f$., 73 fn 41, 74 fn 44, 75, 81f. 81 fn56, 82 fn57, 83 f.

Tamil (script) $59,60 \mathrm{fn} 4,62,63 \mathrm{fn} 8,66$

Tamilian Grantha (script) $\quad 59,59 \mathrm{fn} 1,60$ fn4, 61f.

Telugu (language) $62,80 \mathrm{fn} 55$

Telugu (script) $60 \mathrm{fn} 4,62,62 \mathrm{fn} 6,80 \mathrm{fn} 55$ 